\title{
Multinucleon transfer reactions and proton transfer channels
}

Tea Mijatović ${ }^{1, *}$, Suzana Szilner ${ }^{1}$, Lorenzo Corradi ${ }^{2}$, Franco Galtarossa $^{2}$, Samuel Bakes ${ }^{3,4}$, Daniele Brugnara ${ }^{2}$, Gabriele

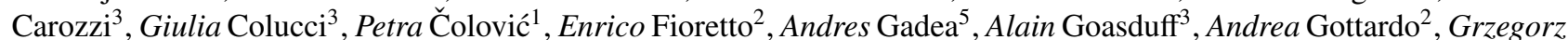
Jaworski $^{6}$, Deša Jelavić Malenica ${ }^{1}$, Tommaso Marchi ${ }^{2}$, Daniele Mengoni ${ }^{3}$, Matko Milin ${ }^{7}$, Giovanna Montagnoli ${ }^{3}$, Deni Nurkić $^{7}$, Giovanni Pollarolo ${ }^{8}$, Romana Popočovski ${ }^{1}$, Fernando Scarlassara ${ }^{3}$, Marco Siciliano ${ }^{2}$, Neven Soić ${ }^{1}$, Alberto Stefanini $^{2}$, Dmitry Testov ${ }^{2,3}$, Calin $\mathrm{Ur}^{9}$, Jose Javier Valiente - Dobón ${ }^{2}$, Nikola Vukman ${ }^{1}$, and Irene Zanon ${ }^{3}$

${ }^{1}$ Ruđer Bošković Institute, Zagreb, Croatia

${ }^{2}$ INFN - Laboratori Nazionali di Legnaro, Legnaro, Italy

${ }^{3}$ Dipartimento di Fisica, Università di Padova and INFN, Padova, Italy

${ }^{4}$ University of Surrey, Guildford, United Kingdom

${ }^{5}$ IFIC CSIC, Valencia University, Spain

${ }^{6}$ Heavy Ion Laboratory, University of Warsaw, Warsaw, Poland

${ }^{7}$ University of Zagreb, Zagreb, Croatia

${ }^{8}$ Università di Torino, and INFN, Torino, Italy

${ }^{9}$ ELI-NP, Bucharest, Romania

\begin{abstract}
Transfer reactions have always been of great importance for nuclear structure and reaction mechanism studies. So far, in multinucleon transfer studies, proton pickup channels have been completely identified in atomic and mass numbers at energies close to the Coulomb barrier only in few cases. We measured the multinucleon transfer reactions in the ${ }^{40} \mathrm{Ar}+{ }^{208} \mathrm{~Pb}$ system near the Coulomb barrier, by employing the PRISMA magnetic spectrometer. By using the most neutron-rich stable ${ }^{40} \mathrm{Ar}$ beam we could populate, besides neutron pickup and proton stripping channels, also neutron stripping and proton pickup channels. Comparison of cross sections between different systems with the ${ }^{208} \mathrm{~Pb}$ target and with projectiles going from neutron-poor to neutron-rich nuclei, as well as between the data and GRAZING calculations, was carried out. Finally, recent results concerning the measurement of the excitation function from the Coulomb barrier to far below for the ${ }^{92} \mathrm{Mo}+{ }^{54} \mathrm{Fe}$ system, where both proton stripping and pickup channels were populated with similar strength, will be discussed.
\end{abstract}

\section{Introduction}

Among the quasi-elastic processes of particular relevance are the ones related to the transfer of nucleon pairs. This type of reactions is perceived as a very promising tool for the study of correlations via simultaneous comparison of $\pm \mathrm{nn}, \pm \mathrm{pp}$ and $\pm \mathrm{np}$ pairs. They are especially advantageous since one could envisage the exchange of multiple nucleon pairs among the reactants for both neutrons and protons.

In studies of pair correlations in heavy-ion collisions several experiments have been performed in the past. In these reactions the signature of correlations is an enhancement of the cross section that, experimentally, may be estimated from the comparison of the measured two-particle transfer probability (or cross section) with the prediction of models using uncorrelated states. Unfortunately the experimental evidence was marred by the fact that almost all existing studies involved inclusive cross sections at energies higher than the Coulomb barrier and at angles forward of the grazing where the reaction mechanism is more complex due to the interplay between nuclear and Coulomb trajectories. Therefore, it is still an open question how the

*e-mail: tea.mijatovic@irb.hr transfer of a nucleon pairs and the correlations affect the quasi-elastic processes.

\section{Multinucleon transfer reactions above the barrier}

In the quasi-elastic regime the mass and charge distributions of transfer products are governed by optimum $Q$ value considerations and transfer form factors. For nuclei close to the stability line, these optimum $Q$-value arguments favor the neutron pickup and the proton stripping channels (pickup and stripping are referring to the lighter reaction fragment) [1]. This is the reason why multinucleon transfer reactions have been used as a competitive tool for the production of neutron-rich nuclei in the vicinity of the light partner [2-8]. This is presented in the Fig. 1 that shows the measured cross sections for the stable open shell ${ }^{58} \mathrm{Ni}$ and closed shell ${ }^{40} \mathrm{Ca}+{ }^{208} \mathrm{~Pb}$ systems where the reaction mechanism strongly favors the proton stripping and neutron pickup channels. It is also evident how the transfer flux changes (shifts) with the use of the neutronrich (stable) projectiles like ${ }^{40} \mathrm{Ar}$ [9]. In particular one sees how proton pickup channels open up. Even if these proton 

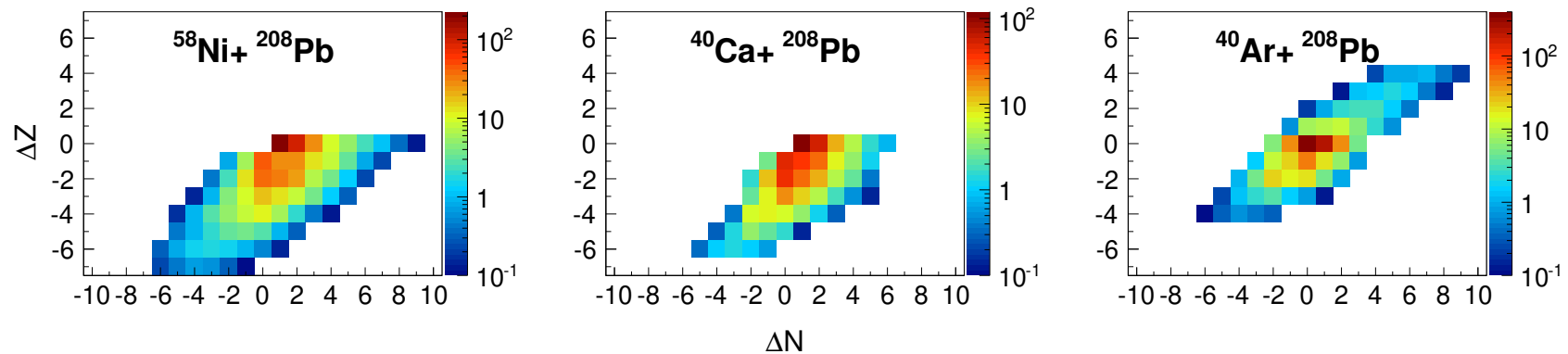

Figure 1. Angle and energy integrated total cross section for ${ }^{40} \mathrm{Ar},{ }^{40} \mathrm{Ca}$, and ${ }^{58} \mathrm{Ni}$ projectiles on the ${ }^{208} \mathrm{~Pb}$ target, at energies $\mathrm{E}_{\mathrm{lab}}=6.4$, 6.2 , and $6 \mathrm{MeV} / \mathrm{A}$, respectively. The cross section values of the elastic (+inelastic) channel have been scaled down by a factor 100 to better display the behavior of the transfer channels [9].

pickup channels have lower cross sections than the proton stripping ones, the observed $Z-N$ distribution turns out to be more symmetric.

Figure 2 shows the data compared with the calculations performed with the GRAZING code [10] for ${ }^{40} \mathrm{Ar}$, ${ }^{40} \mathrm{Ca}$, and ${ }^{58} \mathrm{Ni}$ projectiles on the ${ }^{208} \mathrm{~Pb}$ target [9]. In all systems GRAZING well describes the one nucleon transfer channels. For other pure neutron transfer channels the total cross section is also very well reproduced, which is particularly true for neutron pickup channels. When neutron "stripping" channels are involved, we have to keep in mind that the evaporation of neutrons plays a very important role. Deviations between experimental data and calculations are more marked for channels involving the transfer of many protons. This fact has been discussed in previous publications where experimental cross sections were compared with different semi-classical models in order to see if the addition of new modes, in particular the transfer of a pair of nucleons (both neutrons and protons), may be justified [11]. The inclusion of these pair-transfer modes may be essential. However, we cannot rule out the contribution from deep-inelastic processes. The deviations between experimental data and calculations are more pronounced in the proton pickup sector, in particular when neutron pickup channels are involved, where the contributions from the deep-inelastic collision may be very substantial.

The pure neutron transfer channels have major contribution close to the optimum $Q$-value $(\sim 0 \mathrm{MeV})$, typical of the quasi-elastic regime, with an increasing strength for large energy losses as more neutrons are transferred. For the transfers of more particles, one sees a widening of the experimental $Q$-value and the appearance of the large energy losses. This contribution of the large energy loss components is more pronounced for the proton pickup channels, and when more nucleons are transferred. Such behaviour may indicate that processes more complex than the direct transfer dominate the observed yields. These large energy losses may lead to neutron evaporation which plays a major role in the final mass distribution. Therefore, these additional processes complicate the theoretical calculations even when not considering correlations. Much more work is needed to properly understand, both theoret- ically and experimentally, the behavior of proton transfer channels, in particular the proton pickup ones.

A definite dominance of the proton pickup and neutron stripping channels in the distribution of the transfer flux is predicted to occur with an additional increase of the neutron excess in projectile. Such situation leads to the population of neutron-rich nuclei in the corresponding heavy partner. However, one has to keep in mind that the primary yield can be influenced by secondary processes that generally shift the mass distributions toward lower values.

\section{Multinucleon transfer reactions below the barrier}

At energies closer and well below the Coulomb barrier the contribution of different channels and secondary effects is much lower, which is beneficial to better understanding of proton transfer channels. The interacting nuclei are only slightly influenced by the nuclear potential and $Q$ values are restricted to few $\mathrm{MeV}$ for the open transfer channels. These conditions diminish the complexity of coupled channel calculations, since one needs to take into account only few populated states, and information may be extracted on the nucleon-nucleon correlations.

Measurement itself is more complicated, and significant progress has been made by performing the experiments in inverse kinematics [12]. In this way ions are forward focused which results in high detection efficiency and they have high kinetic energy which is important for energy and therefore mass resolution.

Recently, several systems have been measured with the large solid angle spectrometer PRISMA [13] for the study of nucleon-nucleon correlations [14-16]. PRISMA has a position-sensitive micro-channel plate detector placed at the entrance of the spectrometer, providing a start signal for time-of-flight measurements and bi-dimensional position signals. Ions pass through the optical elements of the spectrometer (a quadrupole and a dipole) and enter a focal plane detector which is made of a parallel plate detector of multi-wire type, providing timing and bidimensional position signals with resolutions similar to the entrance detector. Located at the end of the focal plane is an array of a transverse field multi-parametric ionization chambers (IC), providing nuclear charge via energy loss $(\Delta \mathrm{E})$ and 


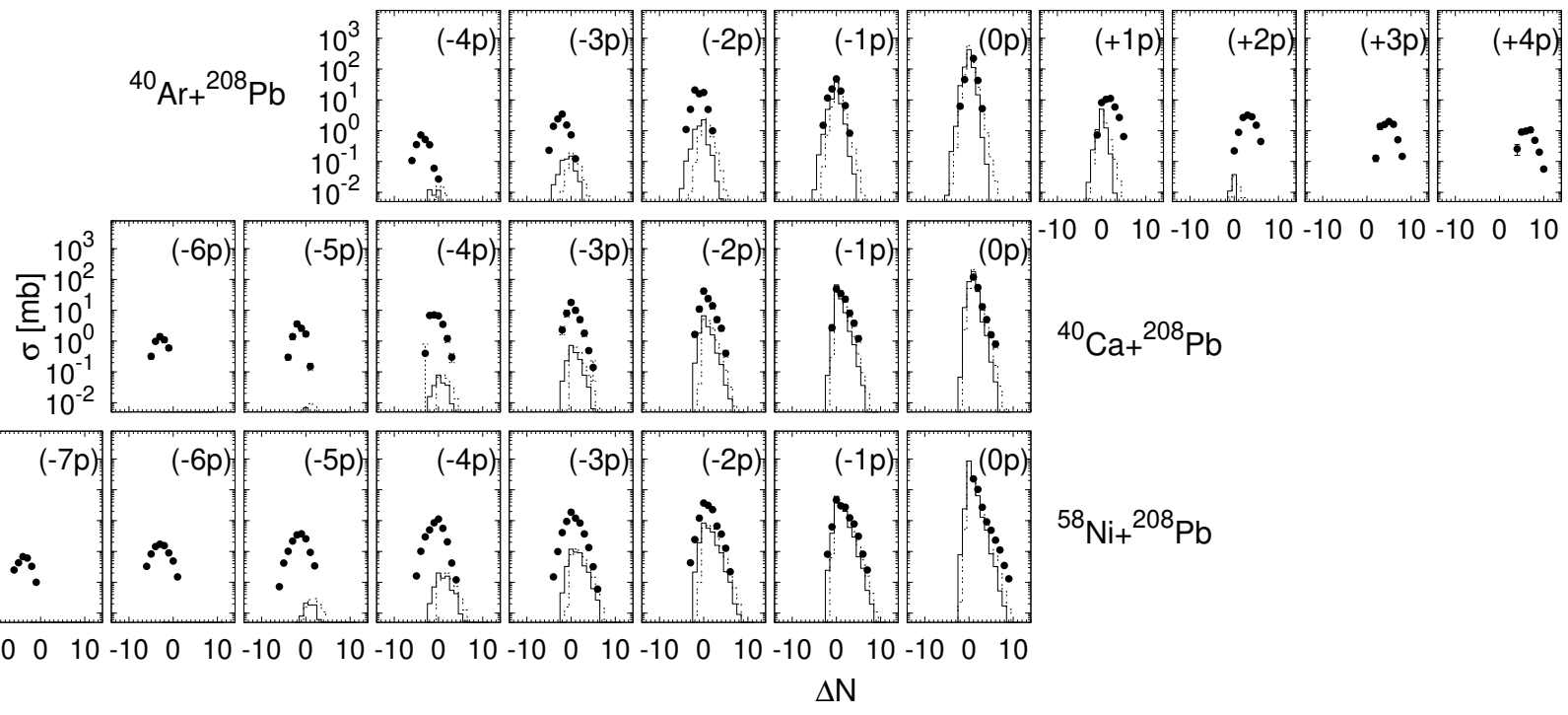

Figure 2. Total experimental cross section for ${ }^{40} \mathrm{Ar},{ }^{40} \mathrm{Ca}$, and ${ }^{58} \mathrm{Ni}$ induced reactions on the ${ }^{208} \mathrm{~Pb}$ target, at beam energies $\mathrm{E}_{\mathrm{lab}}=6.4,6.2$, and $6 \mathrm{MeV} / \mathrm{A}$, respectively (points), and the GRAZING calculations with (solid line) and without (dashed line) neutron evaporation [9].

total energy (E) measurements. The detector system gives all the necessary information for the complete ion identification, which is performed via an event-by-event reconstruction of the trajectory inside the magnetic elements.

Making use of inverse kinematics, target recoils have been detected in multinucleon transfer reactions for the ${ }^{96} \mathrm{Zr}+{ }^{40} \mathrm{Ca}$ (closed shell) [14] and ${ }^{116} \mathrm{Sn}+{ }^{60} \mathrm{Ni}$ (super-fluid nuclei) $[15,16]$ systems. An excitation function has been obtained from the Coulomb barrier to $20-25 \%$ below. This type of experiments is best represented with transfer probabilities, defined as the ratio of the transfer yield over the quasi-elastic one, plotted as a function of distance of closest approach for a Coulomb trajectory, that takes into account both energy and angle.

In the proton sector the experimental data are very scarce. Proton transfer channels are in fact more difficult to measure below the barrier since their cross sections drop off more rapidly than those of neutron transfer channels. Therefore, the proton transfer processes in heavy-ion collisions are much less understood than the ones of neutrons, since large modification in the trajectories of entrance and exit channels are involved due to the modification of the Coulomb field. The single particle level density for protons is less studied than the one of neutrons and the corresponding single-particle form factors are less known (even the one-proton transfer cross sections are not very well described in the DWBA, in fact this theory predicts angular distributions that are shifted in comparison with the data). Moreover, transfer probabilities for protons are generally smaller at the same distances of closest approach. For this reason most of previous works were limited to near-barrier energies and to small values of distances of closest approach, where a non negligible overlap with the absorption region can significantly affect the interpretation of the transfer probabilities and therefore the extracted enhancement coefficients.
We recently made an experiment dedicated to the study of proton transfer channels below the Coulomb barrier where we expect lower contribution of the secondary processes. We chose the ${ }^{92} \mathrm{Mo}+{ }^{54} \mathrm{Fe}$ system involving protonrich nuclei, where proton pickup and neutron stripping channels on ${ }^{54} \mathrm{Fe}$ become available, together with proton stripping and neutron pickup, following optimum $Q$-value considerations. Open channels can be seen in the Fig. 3, that shows GRAZING calculations for the system.

The ${ }^{92} \mathrm{Mo}+{ }^{54} \mathrm{Fe}$ system was measured from above to below the barrier in inverse kinematics using the PRISMA spectrometer. The high efficiency allowed to achieve sufficient yield to get an excitation function encompassing the Coulomb barrier (not far below Coulomb barrier but significantly below the strong absorption radius). We found that the yields of the pure two proton transfer channels are similar to those of the "alpha" transfer channels whose probability turns out to be much stronger than predicted by a simple phenomenological analysis of the probabilities, assuming an independent nucleon transfer process. However, one has to keep in mind that the cross sections are derived by integrating the whole total kinetic energy distributions, therefore, it is important to investigate how the transfer strength is distributed. This information is crucial to understand to what extent the strong population of the $( \pm 2 p)$ and " $( \pm \alpha)$ " channels can be due to simple reaction mechanism effects or whether nucleon-nucleon correlations play a role.

Therefore, we measured, for the same system, fragment-gamma coincidences. We used a ${ }^{54} \mathrm{Fe}$ beam at the bombarding energy $\mathrm{E}_{\mathrm{lab}}=230 \mathrm{MeV}$ (close to the Coulomb barrier) onto a ${ }^{92}$ Mo target with a thickness of $200 \mu \mathrm{g} / \mathrm{cm}^{2}$. Beam-like products were detected with the PRISMA spectrometer placed at $\theta_{\mathrm{lab}}=62^{\circ}$ and $70^{\circ}$, where Fe-like scattered ions have kinetic energies $\sim 2 \mathrm{MeV} / \mathrm{A}$, sufficient to obtain $A$ and $Z$ separation. These angles, close to the grazing, are chosen in order to have a sufficient 


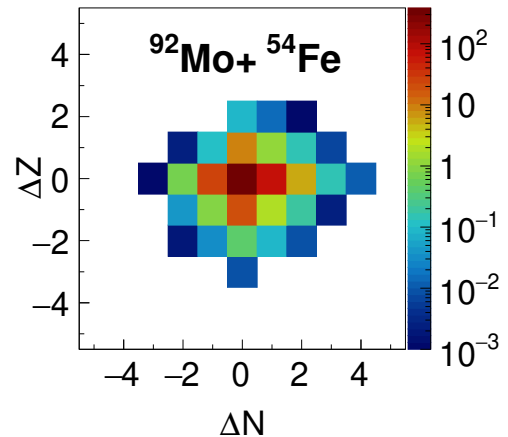

Figure 3. GRAZING calculated cross section for the target-like products in the collision of ${ }^{92} \mathrm{Mo}$ on ${ }^{54} \mathrm{Fe}$ at $370 \mathrm{MeV}$. Relatively symmetric population of neutron and proton pickup and stripping channels is obtained.

transfer yield under the condition that the relative motion trajectories are weakly affected by the nuclear components of the interaction. In this condition the transfer yields are coming from impact parameters leading to large distance of closest approach (where the transfer form factors are of exponential character with their tail governed by the binding energy of the involved single particle states) and are not affected by the absorption.

To measure electromagnetic transitions we used the new array of six 2" $\times 2 " \mathrm{LaBr}_{3} \gamma$ detectors, installed on the top platform of the PRISMA scattering chamber. Each detector was located at $7 \mathrm{~cm}$ from the target and at $45^{\circ}$ with respect to the reaction plane. In this way the intrinsic efficiency for $1.3 \mathrm{MeV} \gamma$-ray was $\sim 1.3 \%$. Tests have been performed with different $\gamma$ sources, getting a timing resolution $\sim 450 \mathrm{ps}$ and an energy resolution $\sim 25-30 \mathrm{keV}$ (for $\sim 1 \mathrm{MeV} \gamma$-ray). This energy resolution is sufficient to separate various transitions, which should allow us to measure the strength distributions.

Data analysis is under-way. Doppler corrected $\gamma$ spectrum for ${ }^{54} \mathrm{Fe}$ shows the strong population of $2^{+}$and $4^{+}$states. Similar approach will be used to extract the strength distributions for different isotopes.

PRISMA also allows to measure differential cross sections by integrating events in steps of $\sim 1.5^{\circ}$ in the reaction plane. This will be used to complement the transfer probabilities obtained via the excitation function previously measured in inverse kinematics. The probabilities obtained via excitation function and angular distribution should be very similar provided that large energy loss components correspond to a small fraction of the cross sections.

\section{Summary}

Transfer reactions with heavy ions are a powerful tool to investigate reaction mechanism and structure properties of nuclei. The comparison of systems going from the neutron-poor ${ }^{40} \mathrm{Ca}$ and ${ }^{58} \mathrm{Ni}$ to the neutron-rich ${ }^{40} \mathrm{Ar}$ projectiles on the ${ }^{208} \mathrm{~Pb}$ target shows how the population trend evolves from proton stripping and neutron pickup to the opposite direction. Such processes are relevant for the production of neutron-rich target-like nuclei and high resolution fragment- $\gamma$ studies are needed to improve the predictability of theoretical models.

Microscopic calculations are still challenging for proton transfer channels. Even if it is expected that at the energies below the Coulomb barrier the energy distribution (TKEL) will become narrower and will concentrate in the single state, the fragment- $\gamma$ coincidences will be crucial to determine which states are dominantly populated, and thus to define the correct phase space used in the calculations.

\section{Acknowledgments}

This work has been supported in part by the Croatian Science Foundation under project no. 7194 and in part under project no. IP-2018-01-1257.

\section{References}

[1] L. Corradi, G. Pollarolo, and S. Szilner, J. Phys. G: Nucl. Part. Phys. 36, 113101 (2009).

[2] S. Lunardi et al., Phys. Rev. C 76, 034303 (2007).

[3] J. J. Valiente-Dobón et al., Phys. Rev. Lett. 102, 242502 (2009).

[4] S. Szilner et al., Phys. Rev. C 84, 014325 (2011).

[5] S. Szilner et al., Phys. Rev. C 87, 054322 (2013).

[6] R. Chapman et al., Phys. Rev. C 93, 044318 (2016).

[7] A. Vogt et al., Phys. Rev. C 93, 054325 (2016).

[8] J. Litzinger et al., Phys. Rev. C 97, 044323 (2018).

[9] T. Mijatović et al., Phys. Rev. C 94, 064616 (2016).

[10] A. Winther, Nucl. Phys. A 572, 191(1994); A. Winther, Nucl. Phys. A 594, 203 (1995); program GRAZING http://www.to.infn.it/ nanni/grazing

[11] S. Szilner et al., Phys. Rev. C 71, 044610 (2005).

[12] F. Galtarossa et al.. Phys. Rev. C 97, 054606 (2018).

[13] S. Szilner et al., Phys. Rev. C 76, 024604 (2007).

[14] L. Corradi et al., Phys. Rev. C 84, 034603 (2011).

[15] D. Montanari et al., Phys. Rev. Lett. 113, 052501 (2014).

[16] D. Montanari et al., Phys. Rev. C 93, 054623 (2016). 